\title{
Programa Nacional de Humanização: implantação na unidade de terapia intensiva em um hospital público de uma cidade do interior de Minas
} Gerais

\author{
DOI: 10.47224/revista master.v6i11.112
}

\author{
Danilo Faria de Moura \\ Emanuel Soares Ponciano \\ Vinicius Bruno Petris Valentim Cortes \\ Lilian Tae Hamada
}

e-mail: viniciusbruno-@hotmail.com

\section{Resumo}

O PNH (Programa Nacional de Humanização) foi criado com o intuito de oferecer acolhimento a todos os pacientes que utilizam o SUS (Sistema Único de Saúde). Sabe-se que nas UTIs (Unidade de Terapia Intensiva) os profissionais de saúde enfrentam situações de grande estresse ligadas ao estado grave e delicado de seus pacientes. Utilizando a metodologia do Arco de Maguerez objetivou-se evidenciar a presença das diretrizes propostas pelo PNH na UTI em um hospital público de uma cidade do interior de Minas Gerais. A pesquisa bibliográfica deu o suporte para a fundamentação teórica e a escolha da bibliografia incorpora consistência à temática. A partir de um diagnóstico foram levantadas possíveis soluções para acolhimento de pacientes, familiares e profissionais da saúde envolvidos nessa empreitada. Observou-se que a situação de exposição à COVID-19 e o estado alarmante de pandemia conduzem os profissionais da saúde para um estado de esgotamento e medo. Em virtude dessa pandemia, e, consequentemente, o aumento de trabalho nas UTIs, não se fez possível a aplicação à realidade das hipóteses de solução levantadas.

Palavras-chave: Programa Nacional de Humanização; Treinamento hospitalar; Unidade de Terapia Intensiva.

\begin{abstract}
The PNH (National Humanization Program) was created with the aim of offering care to all or any patients who use the SUS (Unified Health System). It's known that within the ICUs (Intensive Care Unit) health professionals face great tension hypotheses to the intense and delicate condition of their patients. Using the Arco de Maguerez methodology, the target was to evidence the presence of the proposals by the PNH within the ICU during a public hospital during a city within the interior of Minas Gerais. The bibliographic research supported the theoretical foundation and therefore the choice of bibliography incorporates consistency to the theme. From a diagnosis, possible solutions were raised for the reception of patients, relations and health professionals involved during this endeavor. Note that things of exposure to COVID-19 and therefore the alarming state of the pandemic drive healthcare professionals into a state of exhaustion and fear. Due to this pandemic, and, consequently, the rise in add the ICUs, it had been impossible to use the answer hypotheses raised to reality.
\end{abstract}

Keywords: National Humanization Program; Hospital training; Intensive Care Units.

De acordo com Ministério da Saúde através da portaria no 881, de 19/06/2001, no âmbito do Sistema Único de Saúde (SUS) foi criado o PNH (Programa Nacional de Humanização) para a UTI (Unidade de Terapia Intensiva). O programa propõe que haja processos de discussão e implementação de projetos de humanização do atendimento à saúde e de melhoria da qualidade do vínculo estabelecido entre o profissional de saúde, pacientes e familiares.

O presente estudo é resultante das atividades realizadas no Curso Tecnológico Superior em Gestão de Recursos Humanos. Sua elaboração se deu por meio da Metodologia da Problematização com o Arco de Maguerez analisando sobre os aspectos de 
treinamento e desenvolvimento para a implantação de Programa Nacional de Humanização na UTI em um hospital público no município de Araguari/MG. A metodologia que deu suporte para a fundamentação teórica foi a pesquisa bibliográfica, sendo a eleição da bibliografia consistente com a temática.

Tem-se como principal objetivo analisar e evidenciar a presença das diretrizes propostas pelo PNH na UTI e, a partir desse diagnóstico, propor soluções para que estejam mais latentes, seja por treinamento de aprofundamento ou de educação continuada.

Segundo Chiavenato (2009, p. 41), "treinamento consiste em um processo educacional que é aplicado de maneira sistemática e organizada e pelo qual as pessoas adquirem conhecimentos, atitudes e habilidades em função de objetivos definidos." O treinamento é, portanto, se bem definido e estruturado, um processo educacional que pode gerar mudanças de comportamento. Seu conteúdo pode incluir transmissão de informações e desenvolvimento de habilidades, atividades e conceitos.

O hospital em destaque nesse estudo é um órgão público/filantrópico que atua há mais de 50 anos na saúde do município de Araguari com atendimento de consultas, internações, intensivistas, entre outros. Em 2011 um marco para o referido hospital foi a concepção de um centro médico que possui atendimento misto, particular e gratuito (SUS), sendo um posto de referência em clínica médica de diversas especialidades para Araguari e cidades adjacentes.

Com os acontecimentos no mundo, decorrentes do surgimento de uma nova doença, a COVID-19, notase que equipes de enfermagem do setor de UTI nos hospitais estão encaram alguns problemas relacionados ao enfrentamento desta nova doença. Entre eles está o de não saber totalmente como enfrentar um cenário epidemiológico inédito, o qual ainda não era conhecido por meio de qualquer tipo de literatura cientifica, além de possui sintomas iniciais muito parecidos com outras doenças como gripes, infecções respiratórias, inflamação de garganta, as quais a equipe de atendimento à saúde possui certa familiaridade.

Justifica-se fazer valer as premissas propostas pelo Programa Nacional de Humanização na UTI para o bem da saúde emocional e mental dos envolvidos no tratamento de condições mais críticas de doenças.
Este programa de humanização foi criado com o intuito de dar um acolhimento a todos os pacientes que utilizam o SUS e ao paciente dentro da unidade de tratamento intensivo, pois sabe-se que dentro da UTI é necessário que o paciente tenha um cuidado contínuo e ininterrupto pela equipe de profissionais.

Os problemas, posteriormente aqui listados, estão relacionados com a vivência da equipe de enfermagem na UTI de um hospital público, composta por enfermeiros e técnicos em enfermagem. Entre os problemas que serão apresentados, um deles destaca-se pela não utilização ao Programa Nacional de Humanização e pelo pouco acolhimento apropriado ao paciente em estado grave ou cuidado intensivo, aos familiares e até mesmo aos colegas de trabalho.

\section{IDENTIFICAÇÃO DA PROBLEMÁTICA}

O problema apresentado neste estudo ficou evidenciado a partir da identificação da falta de treinamentos voltados para o desenvolvimento de competências específicas para lidar com fatores emocionais e psíquicos de atitudes mais humanizadas aos pacientes da UTI e seus familiares. Vale ressaltar que o paciente dentro de uma unidade de terapia intensiva, seja ele um recém-nascido, criança, adolescente, jovem, adulto ou idoso, vivência sentimentos de vulnerabilidade, angústia e medo, os quais remetem a um intenso sofrimento emocional, psíquico e físico. A dificuldade de verbalizar, o medo do desconhecido e a dor física muitas vezes impedem a comunicação entre a pessoa enferma e o profissional.

A ausência de treinamentos para o desenvolvimento de competências humanas é um agravante em várias instituições, e quando essas são do segmento hospitalar se torna ainda mais grave.

De acordo com o Conselho Federal e os Conselhos Regionais de Enfermagem, a carga horária média de um enfermeiro é de 12 horas podendo chegar a 24 horas. Já para os técnicos em enfermagem, a legislação determina que a carga horária máxima é de 24 horas. É importante destacar que esses profissionais geralmente trabalham em mais de um local, como relatado em entrevistas feitas durante a pesquisa com alguns deles.

Humanizar é tornar-se humano, humanar-se. Tornando benévolo, afável, tratável, humano. Fazer 
adquirir hábitos sociais polidos; civilizar. A partir deste conceito, é importante abordar a humanização em UTI, pois é um local destinado a um atendimento mais especializado e ininterruptos do cuidado da enfermagem.

\section{COMPREENSÃO DA REALIDADE}

Observando a realidade do hospital, evidenciou-se que a UTI (unidade de terapia intensiva) é um ambiente que apresenta mais tensão e requer mais dedicação e controle emocional dos profissionais que ali atuam:

A unidade de terapia intensiva (UTI) atende à demanda de pacientes que necessitam de cuidados de alta complexidade, prestados por profissionais qualificados e capacitados para desenvolverem a prática em um ambiente altamente tecnológico. (...) Tratase de um local que possui algumas características próprias, como ênfase no conhecimento técnico-científico e na tecnologia, a fim de manter o ser humano vivo; presença constante da morte; ansiedade, tanto dos sujeitos hospitalizados quanto dos familiares e trabalhadores de saúde; rotinas, muitas vezes rígidas $e$ inflexíveis; e rapidez de ação no atendimento, além de um ambiente estressante em razão de ausência de controle de iluminação natural, falta de privacidade e presença de ruídos. (MANDETTA; SOUZA, 2020, p. 65)

Deste modo, entende-se que ainda há o contexto biomédico cuja orientação e cuidado é voltado totalmente para o paciente, a patologia e os procedimentos técnicos em detrimento dos sentimentos, receios dos pacientes e de seus familiares e a forma como vivenciam a saúde-doença.

Frente ao exposto, elencou-se os possíveis fatores imediatos e, também, os condicionantes maiores, associados ao problema destacado.

Possíveis fatores imediatos: ausência de controle emocional entre profissional e paciente, falta de EPIs para os profissionais da saúde que atuam no setor e ausência de relacionamento interpessoal no processo terapêutico.

Possíveis condicionantes maiores: ausência de políticas públicas específicas, falta do suporte necessário para profissionais da enfermagem, sobrecarga na rotina de trabalho e inexperiência de alguns profissionais decorrentes de déficits em sua formação.

A reflexão sobre os fatores imediatos e os condicionantes maiores, associados ao problema levou a destacar alguns aspectos essenciais ou pontos-chave: estresse no ambiente de trabalho, adoecimento mental ou físico por conta da rotina de trabalho e ausência do tratamento humanizado na UTI.

De acordo com Silva (2000, p. 4) "cuidar em Unidades Críticas é ato de amor, o qual está vinculado: a motivação, comprometimento, postura ética e moral, características pessoais, familiares e sociais." Isso possibilita refletir que sempre é necessário um cuidado mais humanizado dentro da Unidade de Terapia Intensiva e um acolhimento empático dos profissionais de enfermagem para com o paciente e seus familiares.

\section{APROFUNDAMENTO TEÓRICO}

Nesta etapa do estudo, apresenta-se uma fundamentação teórica e um embasamento a respeito dos pontos-chave apresentados e que, consequentemente, conduziram às hipóteses de solução para o problema eleito.

Esses aspectos foram objetos de uma revisão literária feita a partir de pesquisas realizadas em artigos científicos, livros e por meio de entrevistas com profissionais da área de enfermagem relacionadas ao tema: Implantação das Diretrizes do Programa Nacional de Humanização na UTI.

Segundo o ex-Ministro da Saúde Carlos Cesar Albuquerque (1997), foi criado o Programa Nacional de Humanização Assistência Hospitalar - PNHAH, mas somente no ano de 2001, sob a orientação do então ex-Ministro da Saúde na época José Serra, ele foi instituído pelo Ministério da Saúde, por meio da portaria 881, de 19/06/2001, no âmbito do Sistema Único de Saúde no Brasil.

\section{ESTRESSE NO AMBIENTE DE TRABALHO}

Nos anos recentes, países de todo o mundo vêm debatendo melhores formas e maneiras de como melhorar o ambiente de trabalho nas UTIs, fazendo 
com que sejam um pouco menos estressante a fim de se manter uma boa saúde psicológica e emocional de todos os profissionais envolvidos.

De acordo com análise dos indicadores obtido por meio das entrevistas, constatou-se que o estresse no ambiente de trabalho tem crescido de forma rápida e considerável, tornando-se um risco a própria saúde mental dos profissionais na área de saúde por conta da sobrecarga de trabalho que tanto tem se acumulado nos hospitais. Evidencia-se um cenário de grande envolvimento e sofrimento emocional entre o grupo de enfermeiros e os demais profissionais da área de saúde.

Segundo Lopes e Lautert (2001, p. 145) "o ambiente da UTI é caracterizado por um trabalho que envolve uma grande carga emocional, na qual a vida e morte se misturam, compondo um cenário desgastante e muita das vezes frustrante."

Ainda neste sentido, Coutrin et al. (2003 apud Salicio e Gaiva, 2006, p. 372) afirmam que "os fatores estressantes no trabalho da enfermagem são: lidar com o sofrimento do paciente e da família, o fazer específico da profissão, a necessidade de improvisação, as questões de ordem burocrática, o inter-relacionamento com a equipe e o barulho constante dos aparelhos."

Um fator que contribui para o estresse da equipe de enfermagem na UTI, e consequentemente no atendimento aos pacientes, é a falta de Equipamentos de Proteção Individual (EPI). Em meio a pandemia da COVID-19 esse problema se agravou gradualmente, fazendo com que muitos profissionais da saúde, principalmente enfermeiros, registrem reclamações nos conselhos de classe regionais e federal, ocasionado protestos por todo país e até pedidos de dispensas e exoneração de cargos nos hospitais.

No que diz respeito a paramentação dos EPIs por parte dos profissionais da enfermagem nas UTIs, o Conselho Federal De Enfermagem (COFEN, 2020) afirma que isso tem como característica histórica atual o combate à pandemia do novo corona vírus.

Desta forma os trabalhadores da área da saúde fizeram reivindicações através dos conselhos de suas categorias profissionais para que fossem tomadas providências em prol de suprir a falta de equipamentos nos hospitais, pois eles estavam na linha de frente no combate à pandemia. Assim sendo, a redação do Brasil Atual, traz uma reportagem abordando sobre a criação de uma campanha intitulada Trabalhadoras e Trabalhadores Protegidos Salvam Vidas, da Internacional Serviços Públicos, alertando sobre a situação dos profissionais de saúde.

Flávio Costa do site UOL, apresenta uma situação crítica vivenciada por médicos e enfermeiros na UTI sucateada do Hospital Público 28 de Agosto, um dos principais na cidade de Manaus no estado do Amazonas. Um dos enfermeiros entrevistados nesta reportagem relatou que: "A UTI está sucateada. Nós não temos material. É um kit de EPI (equipamento de proteção individual) por noite. A gente se contamina com sangue, secreção, e não tem outro para trocar."

\section{AUSÊNCIA DE CONTROLE EMOCIONAL ENTRE PROFISSIONAL E PACIENTE}

Hildegard Peplau (1952) criou a teoria de relacionamento interpessoal que tem como principal característica a interação do enfermeiro com o paciente. Sua teoria busca identificar conceitos e princípios que deem suporte às relações interpessoais que se processam na prática da enfermagem no modo que as situações de cuidado se transformaram em experiências de aprendizagem e crescimento pessoal.

Neste sentido, FAVERO (2009, p. 214) ressalta que se associa a compreensão de Jean Watson (1979), criadora da teoria do cuidado pessoal que tem como característica principal a relação entre saúde, doença e comportamento humano. Seu pensamento teórico considera o cuidado efetivo por meio de um relacionamento transpessoal, ou seja, o cuidado que vai além do tempo, espaço e matéria de paciente e profissional para que forme um único elemento em sintonia.

Em síntese SOUZA (2011) explicam que o estudo acerca do cuidado humanizado se dá a partir da humanização no ambiente hospitalar que deve ser vista de uma forma que ultrapasse o mero ato humanitário. Ele requer um olhar para o processo de comunicação interdisciplinar, bem como para a relação entre a equipe, o paciente e a sua família.

A construção de uma nova práxis no espaço interdisciplinar deve ser norteada por princípios éticos e humanitários, por meio de um processo dialógico e reflexivo. O diálogo, no entanto, não significa ouvir o outro, mas também incentivá-lo à participação no contexto aqui apresentado. 
Desse modo, ampliou-se uma visão acerca das possibilidades referentes a relação entre profissional, paciente e seus familiares.

Considerando o problema em estudo, envolvendo o cuidado humano no ambiente hospitalar, em específico na UTI, nota-se que ainda há muito a ser desenvolvido e melhorado no que tange os cuidados com o paciente.

Ao tratar da questão a respeito de relacionamento interpessoal entre enfermeiro-paciente, buscou-se o embasamento a seguir:

Fundamenta-se na comunicação terapêutica em que são indicadas estratégias para se estabelecer o contato com o paciente. O modo como vem ocorrendo a tecnificação do setor saúde contribui para que muitos enfermeiros negligenciem 0 objetivo principal que é o de cuidar e manter um relacionamento que possa proporcionar uma melhora nos cuidados de enfermagem, e assim, obter resultados benéficos que ajudem no tratamento do paciente. A comunicação que ocorre entre enfermeiropaciente deve proceder de forma objetiva e clara, baseada na empatia e no respeito constante a pessoa que necessita de cuidados por parte do enfermeiro. (BERTONE et al., 2007 p. 1)

Segundo Stefanetli (1983, p. 43) "no relacionamento terapêutico com o paciente a enfermeira desenvolve experiências interpessoais para facilitar a mudança de comportamento do mesmo; ajuda-o a expressar suas ideias, seus sentimentos e seu comportamento." Nessa relação o enfermeiro utiliza a si próprio e as técnicas especificadas no trabalho com o paciente para gerar introvisão e alteração comportamental do paciente.

A partir da década de 40, Helena Willis Render, foi a primeira autora a introduzir a ideia de que o relacionamento entre o enfermeiro e o paciente é de um potencial terapêutico muito significativo. Em 1952, a enfermeira Hildegard Peplau revolucionou esse pensamento e a prática da enfermagem focando no cuidado e relacionamento terapêutico de pessoa para pessoa e, desta forma, fez uma contribuição para área da enfermagem psiquiátrica.

Embora no cenário da UTI sejam comuns discussões acerca da assistência e da forma de trabalho dos profissionais de saúde, frequentemente são questionados o modo de atuação desses profissionais, os quais são inúmeras vezes ligados ao modelo biomédico que condiz com a prática criada no século XIX pelo pensamento mecanicista de René Descartes, Galileu Galilei e Isaac Newton.

Deste modo Hudak, Gallo e Benz (1994) apud Barras et al $(2005$, p. 344) sinalizam que "ser paciente em unidade de cuidados intensivos é potencialmente mais amedrontador, mais solitário, mais confuso e, de certa forma mais desumanizante. Somando-se a isso o papel da enfermagem neste ambiente mudaram." O papel é mais tecnológico, mais orientado fisiologicamente, mais intenso e com maior exigência intelectual que antes.

Segundo Barras et al. (2005, p. 342) "a Associação de Medicina Intensiva Brasileira (AMIB) ressalta que humanização é um processo que envolve todos os membros da equipe da UTI." A responsabilidade da equipe se estende para além das intervenções tecnológicas e farmacológicas focalizados no paciente, incluindo avaliação das necessidades dos familiares, grau de satisfação destes sobre os cuidados realizados, além da preservação da integridade do paciente como ser humano.

É evidente que as exigências impulsionam a ação para implantação do cuidado mais humano no ambiente hospitalar e na unidade de tratamento intensivo. A pesquisa teórica, realizada com o objetivo de buscar fundamentação para problema em destaque, com um embasamento mais profundo acerca dos pontoschave levantados, tornou mais explícito que há muito trabalho a ser feito na enfermagem no que tange o cuidado humanizado. Mesmo sabendo que alguns profissionais da enfermagem ainda trazem consigo o modelo biomédico no momento de atender os pacientes nos hospitais, há aqueles que buscam usar o modelo biopsicossocial e visam o paciente como sendo um ser humano e um ser transpessoal, o qual está além do espaço e tempo.

Em síntese, a etapa da teorização se constituiu condição básica para a apresentação das hipóteses de solução dadas ao problema eleito para este presente estudo.

\section{HIPÓTESES DE SOLUÇÃO DO PROBLEMA}

A reflexão dos aspectos desenvolvidos na teorização, realizada com base na literatura por meio de artigos científicos, livros especializados, sites de internet e 
até entrevistas feitas com profissionais da área, possibilitou, nesta etapa, apresentar diversas alternativas que contribuem para a solução do problema colocado em voga no hospital eleito:

a) desenvolver melhorias de habilidades no cuidado humano na UTI, por meio de indicadores (pesquisa de satisfação do paciente, índice de estresse da equipe de trabalho, controle de atestados e afastamentos da equipe envolvida), além de um acompanhamento sistemático realizado pela liderança do setor, juntamente com a gestão de Recursos Humanos;

b) fortalecer o vínculo no processo terapêutico enfermeiro - paciente, contando com a participação ativa de uma equipe multidisciplinar composta por psicólogo e protocolos de altas e perdas de pacientes;

c) elaborar cronograma de treinamento para as equipes de enfermagem focando no relacionamento interpessoal na unidade de terapia intensiva;

d) promover um melhor relacionamento entre $o$ enfermeiro, o paciente e seus familiares durante visitas na UTI, realizando acolhimento e acompanhamento mais personalizado sanando dúvidas sobre o estado do paciente e promovendo práticas integrativas que contribuam para sua melhora;

e) disponibilizar para as equipes de enfermagem os EPIs necessário, suprindo as necessidades de trabalho seguro para todos os envolvidos;

f) ofertar melhores condições de trabalho na UTI, incluindo a jornada de horário.

\section{APLICAÇÃO À REALIDADE}

Em virtude da pandemia que assolou o planeta e que se agrava nas UTIs com o aumento de infectados pela COVID-19, não foi possível realizar a intervenção com uma proposta de aplicação à realidade.

A partir da análise das hipóteses de solução e de uma reflexão acerca dos fatores imediatos e das condicionantes maiores, bem como do contexto no qual se encontra o problema em questão, foi possível eleger algumas contribuições para sua solução. A proposta inicial era, após realização do diagnóstico, intervir na realidade de modo a buscar o atendimento às necessidades da equipe de enfermagem na unidade de terapia intensiva relacionado ao cuidado mais humano e ainda viabilizar a melhora destes profissionais em saber lidar com a triste realidade que vivenciam na UTI. O diagnóstico partiu da averiguação das premissas previstas pelo PNH (Programa Nacional de Humanização), verificando-se lacunas não atendidas pelo hospital em questão.

Propõe-se que, em um momento mais oportuno, os profissionais que atuam nessa unidade recebam treinamento de educação continuada com periodicidade e acompanhamento pelo setor de Recursos Humanos e pela gestão, para que possam aprimorar continuamente sobre essa necessidade.

Independente do cenário, faz-se relevante e urgente a implantação do acompanhamento psicológico dos trabalhadores que atuam no setor para melhor saúde mental e emocional, estabelecendo-se, inclusive, protocolos de acolhimento coletivo e individual para os casos mais agravantes, como óbitos de pacientes.

\section{CONSIDERAÇÕES FINAIS}

Frente a um cenário inédito de combate a um vírus de grande letalidade e contágio rápido, faz-se necessária e de grande relevância a humanização das UTIs, tanto na gestão dos colaboradores que nela trabalham, como no atendimento aos pacientes e familiares dos enfermos. O cenário mundial de saúde entrou em colapso frente a essa demanda, gerando pânico em parte da população e, consequentemente, colocando os profissionais de saúde que atuam na linha de frente à doença em estado alerta total e mais vulneráveis.

A capacitação continuada e acompanhamento psicológico dos trabalhadores das UTIs deve acontecer de forma sistemática em um calendário estabelecido em conjunto com a gestão do setor e a área de Recursos Humanos. A implantação de um programa de acolhimento psicológico deve ser uma prioridade, em paralelo com os cuidados paliativos que acolhem pacientes e seus familiares, pois as perdas maximizam também nos trabalhadores o estresse.

Foi possível, por meio desse estudo, verificar sobre a presença das diretrizes propostas pelo PNH na UTI e eleger hipóteses para as situações de não 
conformidades identificadas, porém não foi realizada a aplicação de fato em virtude da pandemia da COVID-19.

Em pleno cenário de pandemia muitos enfermeiros e profissionais da saúde tem enfrentado grandes questões humanitárias, e uma delas é lidar com a perda de colegas profissionais e os próprios pacientes. Contudo alguns profissionais têm deixado de lado este medo e buscado sempre ajudar o próximo, independente do que aconteça, pois como diz a médica e escritora Ana Claudia Quintana Arantes: "A morte é um dia que vale a pena viver".

\section{REFERÊNCIAS}

BARRAS, Daniela Couto Carvalho; JUSTINA, Anelice Della; BERNARDES, Jane Fonseca de Lacerda; VESPOLI, Fabíola; REBOUÇAS, Ula; CADETE, Matilde Meire Miranda. Processo de humanização e a tecnologia para o paciente internado em uma unidade de terapia intensiva. REME - Rev. Min. Enf., v.9, n.4, p.344-350, 2005.

BERTONE, Tássia Bruschini; RIBEIRO, Ana Paula Sousa; GUIMARÃES, Jacileide. Considerações sobre o relacionamento interpessoal enfermeiro-paciente.

Revista Fafibe [on-line], n.3, 2007. ISSN 1808-6993. Disponível em:

http://www.unifafibe.com.br/revistasonline/arquivo s/revistafafibeonline/sumario/11/19042010141352. pdf. Acesso 28 maio 2020.

\section{BRASIL. MINISTÉRIO DA SAÚDE. Galeria de}

Ministros. Disponível em:

https://www.saude.gov.br/galeria-de-ministros.

Acesso 28 maio 2020.

\section{CHIAVENATO, Idalberto. Treinamento e} desenvolvimento de recursos humanos: como incrementar talentos na empresa. 7.ed. Barueri: Manole, 2009.

COFEN - Conselho Federal de Enfermagem. Mais de cem municípios reduziram para $30 \mathrm{~h}$ a jornada de enfermagem. Disponível em:

http://www.cofen.gov.br/mais-de-cem-municipiosreduziram-para-30h-a-jornada-dos-profissionais-deenfermagem 48298.html. Acesso 28 maio 2020.

COSTA, Flávio. Covid-19: "Falta material para trabalhar na UTI ", diz enfermeiro em Manaus. 2020.
Disponível em:

https://noticias.uol.com.br/saude/ultimasnoticias/redacao/2020/05/01/covid-19-faltamaterial-para-trabalhar-na-uti--diz-enfermeiro-emmanaus.htm. Acesso 30 maio 2020.

FAVERO, Luciane et al. Aplicação da teoria do cuidado transpessoal de Jean Watson: uma década de produção brasileira. Acta paul. enferm., v.22, n.2, p.213-218, 2009. Disponível em:

http://www.scielo.br/scielo.php?script=sci arttext\& pid=S0103-21002009000200016\&lng=en\&nrm=iso.

Acesso 28 maio 2020.

LOPES, M.J.M.; LAUTERT, L. A saúde das trabalhadoras da saúde: algumas questões. In: HHAAG,G.S.;LOPES, M. J.; SCHUCK, J.S.(org). A enfermagem e a saúde dos trabalhadores. Goiânia: $A B$, p. 109-140, 2001.

MANDETTA, M; SOUZA, R. A humanização e o suporte emocional na UTI: equipe, familiares e pacientes. In: Pereira, V. R. A. P. et al. (Org).

Enfermagem em Terapia Intensiva: Práticas e Vivências. 2. ed. Porto Alegre: Artmed, p. 64-79, 2000.

PEPLAU, Hildegard E. The psychiatric nurses' family group. The American journal of nursing, p. 14751477, 1952.

Portaria MS/GM no 881, de 20/06/2001: Institui, no ambito do Sistema Unico de Saúde, o Programa Nacional de Humanização da Assistência Hospitalar PNHAH.

RBA - Rede Brasil Atual. Para profissionais da saúde, falta proteção, sobram riscos e angústia. 2020. Disponível em:

https://www.redebrasilatual.com.br/trabalho/2020/ 04/pesquisa-profissionais-saude-riscos-angustias/. Acesso 30 maio 2020.

SALICIO, D. M. B. S.; GAIVA, M. A. M. O significado de humanização da assistência para enfermeiros que atuam em UTI. Revista Eletrônica de Enfermagem, v. 08, n.03, p. 370-376, 2006. Disponível em: http://www.fen.ufg.br/revista/revista8 3/v8n3a08.h tm. Acesso 28 maio 2020.

SILVA, M.J.P. da. Humanização em UTI. In: CINTRA, E.A.; NISHIDE, V.M.; NUNES, W.A. (Org.). Assistência 
de enfermagem ao paciente crítico. São Paulo:

Atheneu, 2000. p. 3-11.

SOUZA, Ellen Lucy Vale de et al. Uso dos

equipamentos de proteção individual em unidade de terapia intensiva. Revista de Enfermagem

Referência, v.3, n.4, 2011.

STEFANETLI, Maguida Costa. Relacionamento terapêutico enfermeira-paciente. Revista da Escola

de Enfermagem da USP, v. 17, n. 1, p. 39-45, 1983. 